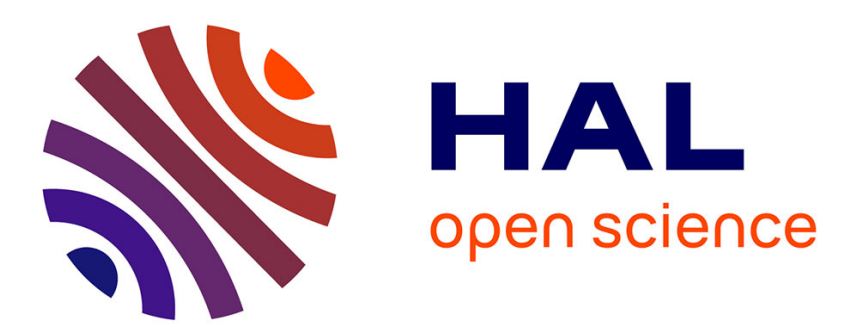

\title{
An image-based computational model of oscillatory flow in the proximal part of tracheobronchial trees
}

\author{
Catalin Feita, Simona Mancini, Diane Perchet, Françoise Prêteux, Marc
}

Thiriet, Laurence Vial

\section{- To cite this version:}

Catalin Feita, Simona Mancini, Diane Perchet, Françoise Prêteux, Marc Thiriet, et al.. An imagebased computational model of oscillatory flow in the proximal part of tracheobronchial trees. Computer Methods in Biomechanics and Biomedical Engineering, 2005, 8(4), pp.279-293. hal-00021505

\section{HAL Id: hal-00021505 https://hal.science/hal-00021505}

Submitted on 25 Oct 2012

HAL is a multi-disciplinary open access archive for the deposit and dissemination of scientific research documents, whether they are published or not. The documents may come from teaching and research institutions in France or abroad, or from public or private research centers.
L'archive ouverte pluridisciplinaire HAL, est destinée au dépôt et à la diffusion de documents scientifiques de niveau recherche, publiés ou non, émanant des établissements d'enseignement et de recherche français ou étrangers, des laboratoires publics ou privés. 


\section{An image-based computational model of oscillatory flow in the proximal part of tracheobronchial trees}

Fetita C..$^{\dagger}$, Mancini S. ${ }^{\ddagger 1}$, Perchet D. ${ }^{\dagger}$, Prêteux F. ${ }^{\dagger}$, Thiriet M. ${ }^{\ddagger 2}$, Vial L. ${ }^{\ddagger}$

† Unité de Projets ARTEMIS, Institut National des Télécommunications, F-91011 Evry, France,

$\ddagger$ INRIA team REO, LJLL, UMR CNRS 7598, Université Pierre et Marie Curie, F-75252 Paris cedex 05 and INRIA, BP 105, F-78153 Le Chesnay Cedex, France.

running title : Oscillatory flow in the tracheobronchial tree

\footnotetext{
${ }^{1}$ now in Laboratoire MAPMO, Université d'Orléans, BP 6759, 45067 Orléans cedex 2

${ }^{2}$ correspondence : Marc.Thiriet@inria.fr
} 
Abstract A computational model of an oscillatory laminar flow of an incompressible Newtonian fluid has been carried out in the proximal part of human tracheobronchial trees, either normal or with a strongly stenosed right main bronchus. After acquisition with a multislice spiral CT, the thoracic images are processed to reconstruct the geometry of the trachea and the six first bronchus generations and to virtually travel inside this duct network. The facetisation associated with the three-dimensional reconstruction of the tracheobronchial tree is improved to get a computationadapted surface triangulation, which leads to a volumic mesh composed of tetrahedra. The Navier-Stokes equations associated with the classical boundary conditions and different values of the flow dimensionless parameters are solved using the finite element method. The airways are supposed to be rigid during rest breathing. The flow distribution among the set of bronchi is determined during the respiratory cycle. Cycle reproducibility and mesh size effects on the numerical results are examined. Helpful qualitative data are provided rather than accurate quantitative results in the context of multimodelling, from image processing to numerical simulations.

Keywords computed tomography, flow distribution, oscillatory flow, tracheobronchial tree, 3D reconstruction.

\section{INTRODUCTION}

Alternatively inhaled and exhaled air flows in the respiratory conduits. The respiratory tract is composed of the upper airways and the tracheobronchial tree. The usual end of the respiratory conduits associated with the ambient air is the nose or, especially during lung function testing, the mouth. The nose, the pharynx and the larynx constitute the upper airways, where the air is warmed and moistened. The upper airways convey a developing three-dimensional flow, whose velocity distribution is not accurately known. For ethical reasons, the image acquisition does not target the whole respiratory tract. Consequently either the upper airways or the thoracic respiratory conduits are reconstructed.

The trachea crosses the superior thoracic aperture and enters the thoracic cage, travelling in the posterior mediastinum. The trachea bifurcates into the right and the left main (or stem) bronchus, the left one branching off at a sharper angle than the right one. Each main bronchus enters in the lung parenchymas through the hilum, with blood vessels of the systemic and pulmonary circulation, lymphatics and nerves, to give birth to the corresponding bronchial tree. The bronchial trees are characterized by branching curved short ducts (non-symmetrical bifurcation, sometimes trifurcation, possible lateral branching). The tracheobronchial tree can be described by a set of generations, generation 0 (G0) being the trachea. The first generations of the tracheobronchial tree, the lobar and segmental bronchi, constitute the proximal airways which are visible by classical imaging techniques. Both the bronchus curvature and branching being non-planar, bronchus paths have to be accurately reconstructed in order to preserve the flow behaviour. The greater the generation number, the narrower the lumen and the smaller the length $L$. In the last generations the ratio between the length and the caliber is equal to about 1 .

The tracheobronchial tree is composed of a succession of bends and branching sites. Hence, the air is conveyed by a developing, three-dimensional, oscillatory flow. The present joint study is a preliminary step toward a computer-aided medical tool for the diagnosis and the aerosol therapy. Delivery and uptake of pharmaceutical drugs by inhalation require the knowledge of the three-dimensional unsteady flow within the airways. Because of the huge between-subject variability in anatomy, medical tools deal with patient-specific geometries. Nowadays, scanning devices are able to model the anatomy of the thoracic airways. Afterwards, numerical flow simulations can be carried out in domains based on the $3 \mathrm{D}$ reconstruction of these airways $[14,3]$.

Sufficiently accurate geometrical models were developed in order to enable virtual endoscopy as well as flow computations, using the the Navier-Stokes equations. The local air flow as well as the flow distribution among the bronchi give important parameters for the medical checkup.

\section{METHOD}

Air flow simulations in models of the tracheobronchial tree require a set of computational tools, from the three-dimensional reconstruction of the surface of the fluid domain to the computational grid generation, and, finally to the Navier-Stokes equation solving.

\section{Geometry of the fluid domains}

Two kinds of tracheobronchial trees are used in the present study, a normal- (M1) and a pathological (M2) model. The normal tracheobronchial model is composed of the trachea and the proximal generations, down to G5, mainly in the left side, and G6 in the right network with some G7 (Fig. 1). Few lateral branches, 


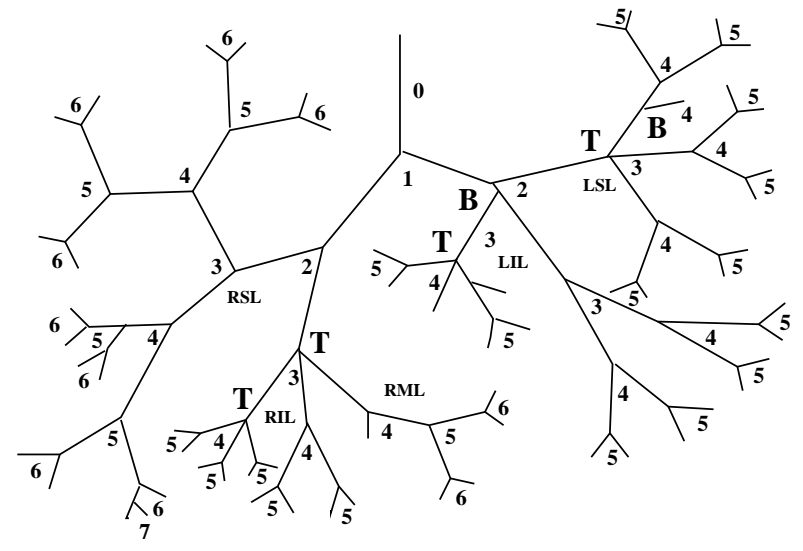

Figure 1: Schematic drawing of the tracheobronchial model M1 with generation number obtained from visualization of the computational model (B: lateral branching, T: trifurcation, RSL: right superior lobe, RML: right middle lobe, RIL: right inferior lobe, LSL: left superior lobe, LIL: left inferior lobe). The right tree part often contains a higher number of generations. It has 32 bronchial ends whereas the left counterpart has 27.

of smaller bore, as well as trifurcations are observed on the visualized mesh of the reconstructed M1 tree. The junction model is quite irregular. The pathological tracheobronchial tree is characterized by a severe stenosis $(\sim 90 \%)$ of the right stem bronchus.

\section{Image acquisition}

Multislice helical volumetric CT (MSSCT) allows fast acquisitions of the whole thorax during a simple breath hold (about $20 \mathrm{~s}$ ), with better longitudinal and transverse spatial resolution. The data set acquired from MSSCT consists of overlapping images along the craniocaudal axis ( $z$ axis) of the body. The longitudinal resolution is determined by the collimator width, pitch, and interpolation algorithm. The present reconstruction interval is equal to $0.6 \mathrm{~mm}$. The transverse resolution, along the $x$ - and $y$ axes, depends on the selected field of view. It is equal to 0.56 and $0.73 \mathrm{~mm}$ for M2 and M1 respectively. The voxels thus are anisotropic.

The 3D reconstruction of the tracheobronchial tree based on MSSCT requires an accurate extraction of the airway morphology from the volumetric image data. The marching cube technique is a generic approach, based on a "voxelhood" analysis, which provides a surface representation of the targeted organ from MSSCT, extracting an implicit surface [15]. But, it imposes a constraint associated with the selected isovalue. The isovalue is easily chosen when the organ structure can be merely differentiated from their image environment by a thresholding operation. In the case of MSSCT images of the thorax, a single threshold fails to extract the inner airway wall due to the gray-level variability related to partial volume effects, noise, and pathologies (stenosis, lung emphysema, fibrosis, Fig. 2). Hence, the segmentation of airway luminae must be treated before any surface mesh representation.
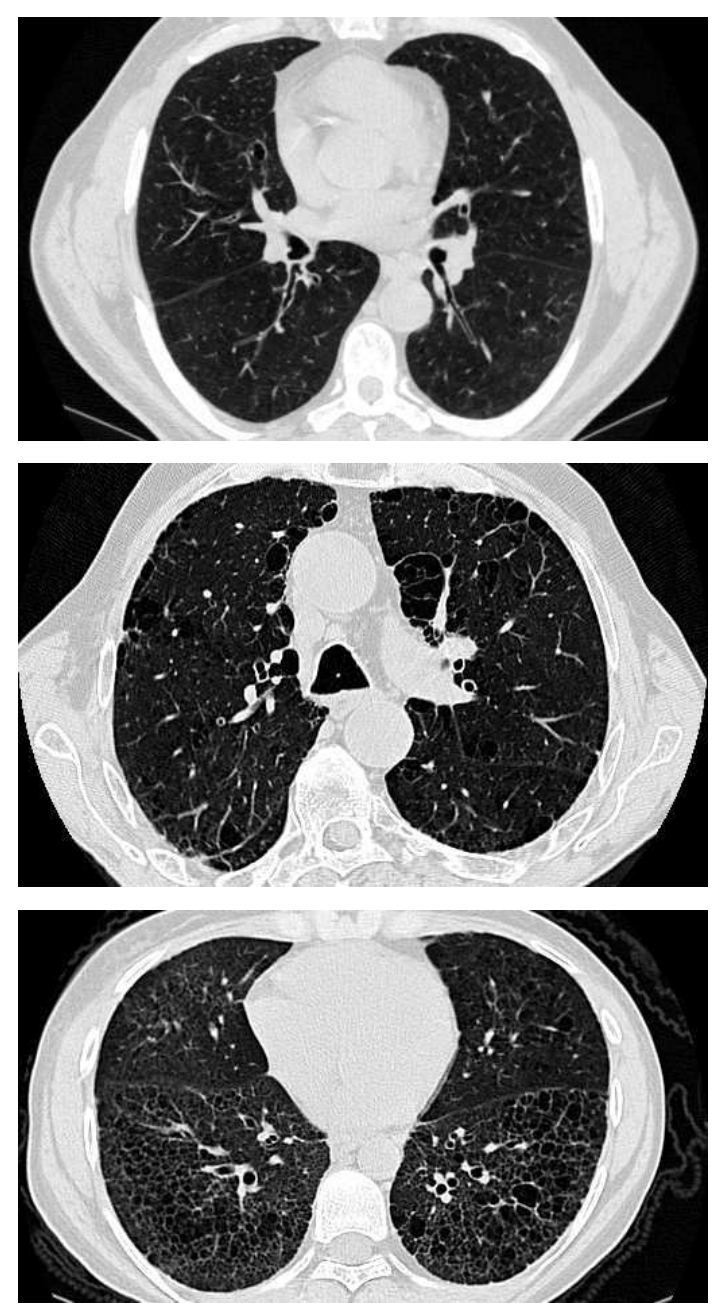

Figure 2: Examples of MSSCT images showing the difficulty of airway segmentation. Top: partial volume effect. Mid: lung emphysema. Bottom: fibrosis.

\section{Image processing}

A specific 3D segmentation approach for the tracheobronchial tree has been developed in order to take into account the MSSCT image characteristics and to overcome the limitations of the existing segmentation techniques. Such approach first identifies the 3D regions in the image volume corresponding to airway luminae (marking step), then performs an energy-based region growing conditional to local intensity and topology constraints (reconstruction step) [6]. 


\section{Airway segmentation}

The marking step is aimed at discriminating the airway luminae from noise and similar zones in the lung parenchyma, and also at providing an extended subset of low-order subdivision segments. The developed marking approach relies on a multiresolution morphological filtering using the SMDC-connection cost operator, defined in the following.

Def. 1 We call $L$-connected a function $f: \Re^{n} \rightarrow \Re$ of connected support, $\operatorname{supp}(f)$, and upper bounded on any bounded subset of $\operatorname{supp}(f)$.

Def. 2 Let $f$ L-connected and $\lambda \in \Re$ a real value. The lower threshold of $f$ at level $\lambda$ is given by :

$$
\mathrm{X}_{f, \lambda}=\{x \in \operatorname{supp}(f), f(x) \leq \lambda\} .
$$

Similarly, the upper threshold of $f$ at level $\lambda$ is defined by :

$$
\check{\mathrm{X}}_{f, \lambda}=\{x \in \operatorname{supp}(f), f(x) \geq \lambda\} .
$$

Not. 1 Let $f$ L-connected and $\lambda \in \Re . \delta_{f, \lambda}$ and $\check{\delta}_{f, \lambda}$ denote the geodesic distance with respect to $\mathrm{X}_{f, \lambda}$ and $\check{\mathrm{X}}_{f, \lambda}$, respectively.

Def. 3 Let $f \leq g$ two L-connected functions of same support. We call connection plateau of $f$ with respect to $g$, at a point $x \in \operatorname{supp}(f)$, the subset :

$$
\begin{gathered}
\mathrm{P}_{f}^{g}(x)=\left\{\lambda \in \Re, \lambda \geq f(x) / \forall y \in \mathrm{X}_{f, \lambda}\right. \text { such that } \\
\left.\delta_{f, \lambda}(x, y)<+\infty \Longrightarrow \check{\delta}_{g, \lambda}<+\infty\right\}
\end{gathered}
$$

Note that $\mathrm{P}_{f}^{g}(x)$ may as well contain an infinity of values, as equal the empty set, as illustrated in FIG. 3a: $\mathrm{P}_{f}^{g}\left(x_{1}\right)=\emptyset$ and $\mathrm{P}_{f}^{g}\left(x_{2}\right)=\emptyset$ (the condition (3) is not met for $y_{1}$ and $y_{2}$, respectively), while $\mathrm{P}_{f}^{g}\left(x_{3}\right)=$ $\left[f\left(x_{3}\right), h\right]$.

Def. 4 Let $f \leq g$ two L-connected functions of same support. We call maximal connection plateau of $f$ with respect to $g$, at a point $x \in \operatorname{supp}(f)$, the set (Fig. 3b):

$$
\mathrm{M}_{f}^{g}(x)= \begin{cases}\sup \left\{\mathrm{P}_{f}^{g}(x)\right\}, & \text { if } \mathrm{P}_{f}^{g}(x) \neq \emptyset \\ f(x), & \text { otherwise }\end{cases}
$$

Note that $\mathrm{M}_{f}^{g}$ is equivalent to the upper-leveling operator of $g$ with respect to $f[19]$.

Def. 5 Let $f$ L-connected. The connection cost of a point $x \in \operatorname{supp}(f)$ to a non-empty subset $\mathrm{Y} \subset \operatorname{supp}(f)$ [21] is defined as:

$$
\begin{aligned}
& \forall x \in \operatorname{supp}(f), \mathcal{C}_{f}(x, Y)= \\
& \begin{cases}\inf \left\{\lambda \in \Re / \delta_{f, \lambda}(x, Y)<+\infty\right\} & \text { if } x \notin Y \\
-\infty, & \text { otherwise. }\end{cases}
\end{aligned}
$$

Def. 6 Let $f \leq g$ two L-connected functions of same support. The sup-constraint connection cost of a point $x \in \operatorname{supp}(f)$ to a non-empty subset $Y \subset \operatorname{supp}(f)$ withrespect to $f$ and $g$ is defined as:

$$
\mathcal{R C}_{f}^{g}(x, Y)= \begin{cases}\inf \left\{\mathcal{C}_{f}(x, Y), \mathrm{M}_{f}^{g}(x)\right\}, & \text { if } x \notin Y \\ f(x), & \text { otherwise }\end{cases}
$$

A comparative illustration of $\mathcal{C}_{f}(., Y)$ and $\mathcal{R C}_{f}^{g}(., Y)$ operators with respect to a subset $Y \subset \operatorname{supp}(f)$ is shown in Fig. 3c and 3d.

If $f$ is interpreted as a topographical relief, where intesity values $f(x)$ represent altitudes, then $\mathcal{R C}_{f}^{g}(., Y)$ is obtained by "flooding" $f$ up to reaching an equilibrium, knowing that $f$ is totaly "permeable" at the level of $Y$ and that the "water" level cannot go beyond $g$.

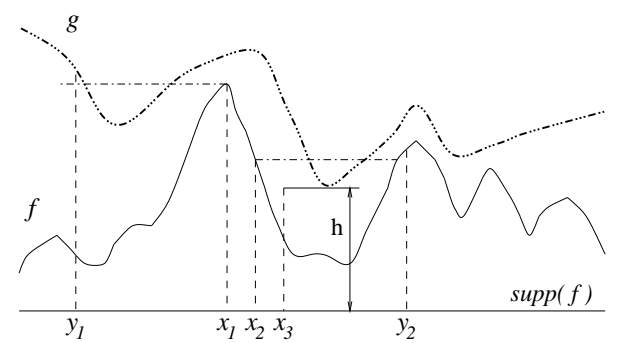

(a) $\mathrm{P}_{f}^{g}(x)$

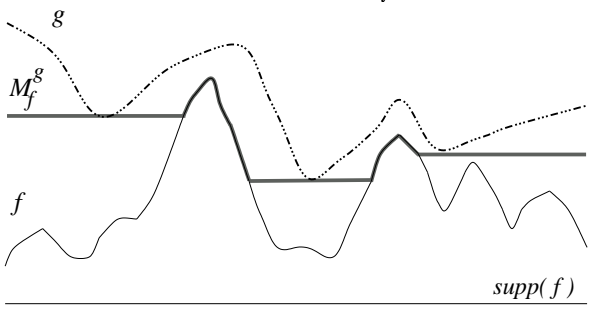

(b) $\mathrm{M}_{f}^{g}$

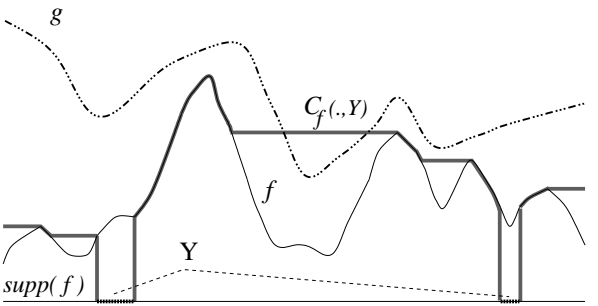

(c) $\mathcal{C}_{f}(., Y)$

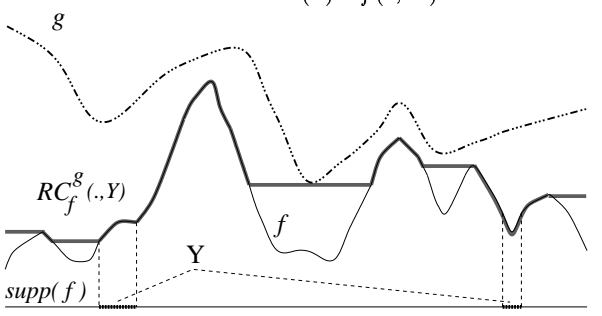

(d) $\mathcal{R C}_{f}^{g}(., Y)$

Figure 3: Definition of the sup-constraint connection cost operator.

Def. 7 Let $f$ L-connected and $n \in \Re$. The selective marking and depth constrained (SMDC)-connection cost of size $n$, of a point $x \in \operatorname{supp}(f)$ to a non-empty subset $\mathrm{Y} \subset \operatorname{supp}(f), \mathcal{R C}_{f}^{n}(., Y)$, is a particular case of $\mathcal{R C}_{f}^{g}(., Y)$, defined as:

$$
\mathcal{R C}_{f}^{n}(., Y)=\mathcal{R C}_{f}^{g_{n}}(., Y),
$$

with

$$
g_{n}=\min \left(f \oplus B_{n}, f \oplus \check{B}_{n}\right),
$$


where $B_{n} \subset \operatorname{supp}(f)$ denotes a causal structuring element of size $n, \check{B}_{n}$ its symmetric with respect to the origin, and $\oplus$ the Minkowski addition.

Considering the previous interpretation of $f$ in terms of topographical relief, $\mathcal{R C}_{f}^{n}$ achieves a controlled relief "flooding", which affects only the "valleys" of size smaller than $n$ and insulated from other valleys of size $n$ or larger. $\forall Y \subset \operatorname{supp}(f)$,

$$
\begin{array}{ll}
\mathcal{R C}_{f}^{0}(x, Y)=f(x), & \forall x \in \operatorname{supp}(f) \\
\mathcal{R C}_{f}^{\infty}(x, Y)=\mathcal{C}_{f}(x, Y), & \forall x \in \operatorname{supp}(f) \backslash\{Y\} \\
\mathcal{R C}_{\psi_{n}}^{N}(., Y)=\mathcal{R C}_{f}^{N}(., Y) & \forall n<N, \psi_{n}=\mathcal{R C}_{f}^{n}(., Y) .
\end{array}
$$

The properties of $\mathcal{R} \mathcal{C}_{f}^{n}$ allows to implement a marking approach in a multiresolution scheme as illustrated on the synthetic data displayed on Fig. 4a. The reference subset $Y$ is considered as the union of the lateral walls of the image volume in order to prevent any connection with the bronchial tree. First, the original data is filtered with $\mathcal{R C}_{f}^{n}$ of size $n$ smaller than the trachea cross-section (automatically computed). The noise and non-bronchial components are leveled-up, whereas the small caliber bronchi remain unaffected due to their connection with the trachea (Fig. 4b). Second, the original data are filtered with $\mathcal{R C}_{f}^{N}$ of size $N$ larger than the trachea, which will result in "flooding" the airway lumen (Fig. 4c). An adaptive thresholding of the difference $\mathcal{R C}_{f}^{N}-\mathcal{R C}_{f}^{n}$ provides the airway marking set (Fig. 4d).

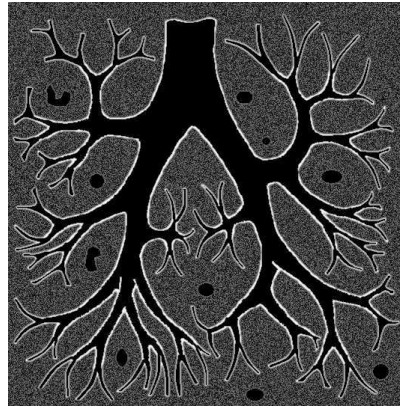

(a) $f$

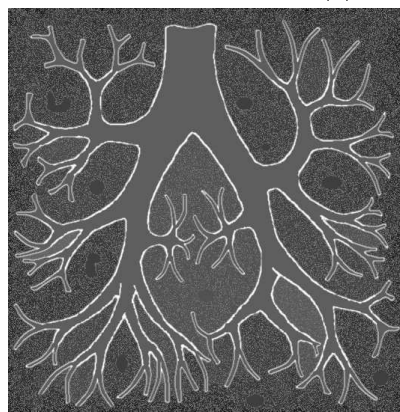

(c) $\mathcal{R C _ { f } ^ { N }}$

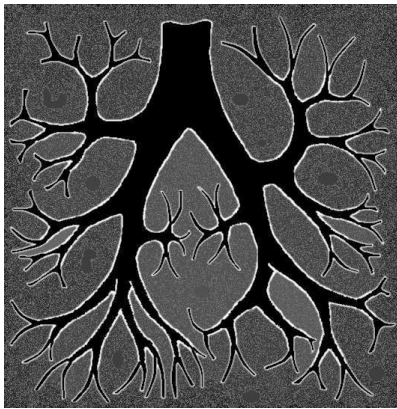

(b) $\mathcal{R C}_{f}^{n}$

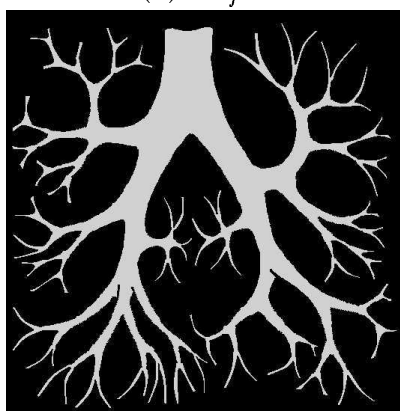

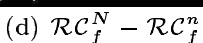

Figure 4: Multiresolution marking scheme.

Fig. 5 shows an example of low-order airways obtained from MSSCT data with the described marking approach.
High-order bronchi are then reconstructed by performing a region growing conditional to local constraints on gradient, topology and image intensity. The propagation condition at a point $x$ on the boundary of loworder airway subset in a given direction $\mathbf{d}$ is described by:

$$
(\mathbf{d} \cdot \nabla f)(x)+\mathcal{T}(x, f, \mathbf{d})-k f(x)>0,
$$

where $\mathcal{T}$ characterizes the local topology with respect to $f$ and $\mathbf{d}$ favoring the propagation inside small caliber bronchi [6], and $k$ is a normalizing parameter, set up according to the MSSCT acquisition protocol. The tracheobronchial tree is segmented up to G6, overcoming the limitations of classic segmentation techniques related either to insufficient accuracy or to lack of robustness (Fig. 5).
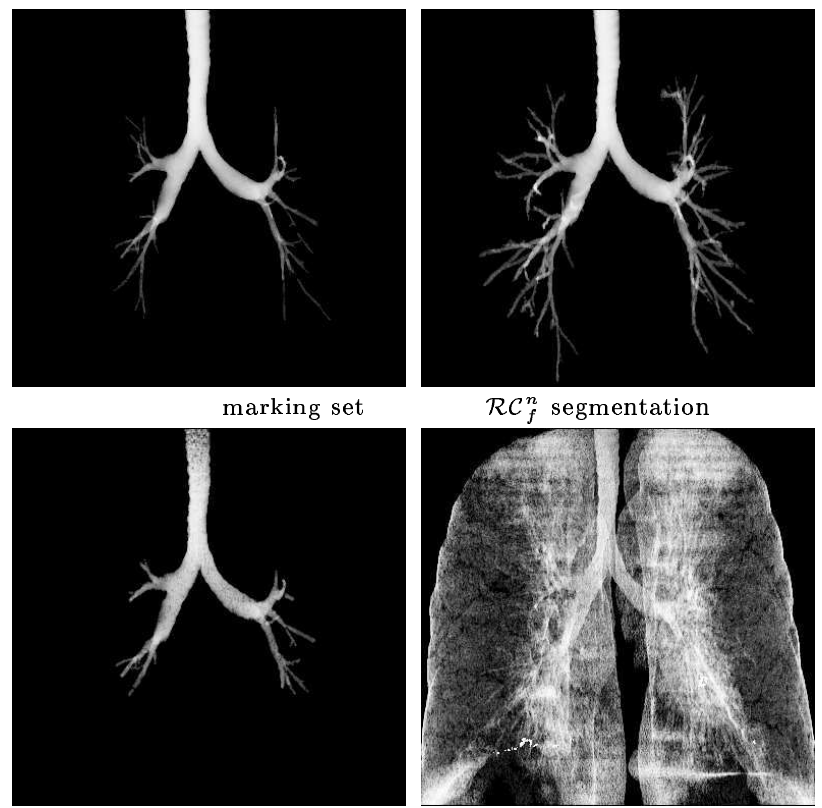

$\mathcal{R C}_{f}^{n}$ segmentation

morphological closing

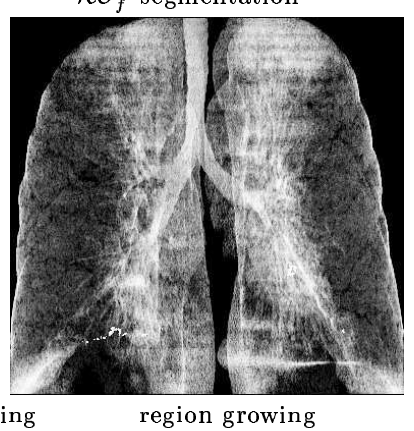

Figure 5: 3D segmentation of the tracheobronchial tree from MSSCT. Top: present approach. Bottom: classic techniques with insufficient accuracy (left) or lack of robustness (right).

\section{Surface extraction}

The segmented bronchial tree is a $3 \mathrm{D}$ binary structure. Extracting a mesh from binary data by using the marching cubes algorithm imposes some additional processing step in order to provide smooth meshes while preserving the topology and the geometry of the binary model (Fig. 6). A solution consists in smoothing the binary data with a Gaussian kernel prior to applying the marching cubes. Conversely, such smoothing will require a tune-up of the isovalue parameter in order to preserve the original caliber of the segmented bronchi. 
Due to the bronchus caliber variation with the generation order, a constant isovalue and a fixed Gaussian kernel cannot achieve the requirements related to mesh smoothing, mostly involving low-order bronchial generations, and to caliber/topology preservation, associated with the high-order generations (Fig. 7).

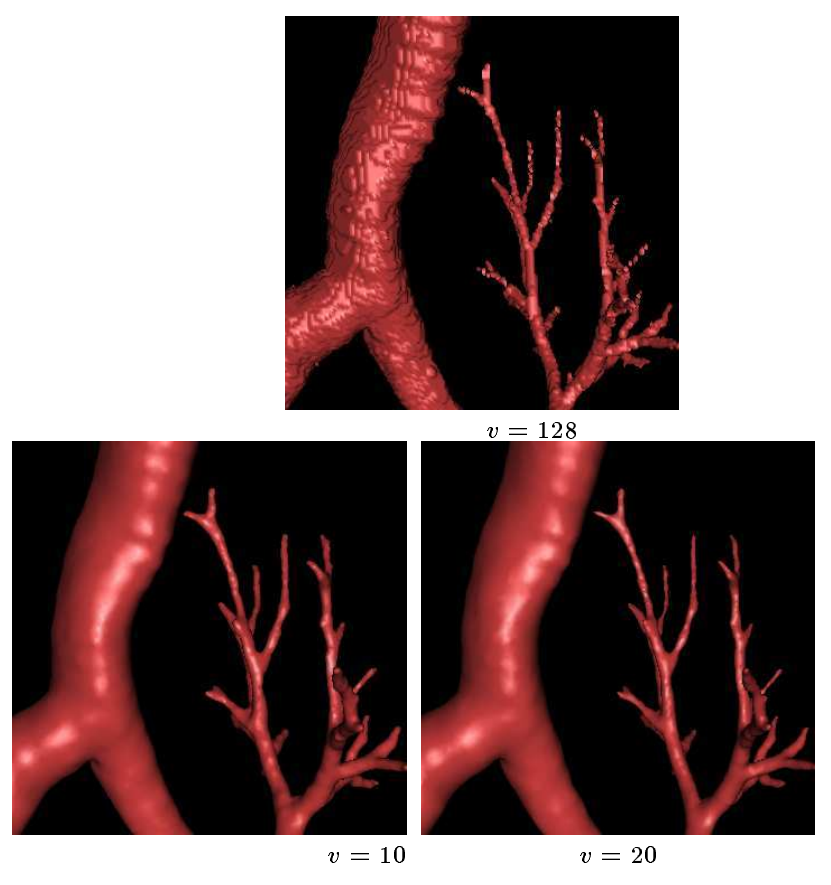

Figure 6: Marching cube-based surface meshing from 3D binary data, different isovalues $v$ providing different bronchial bores. Top: without smoothing, with surface irregularities and/or segment disconnection. Bottom: with Gaussian pre-filtering.

In order to overcome these drawbacks, a marching cubebased adaptive approach has been developed to extract a surface mesh from 3D binary data. The technique adaptively performs both data smoothing and isovalue selection according to the local bronchial caliber. The caliber information is provided by a central axis-based description of the segmented airway morphology.

\section{Airway axis set}

The axes of complex branching structures such as tracheobronchial trees are accurately determined when the duct network geometry and generation hierarchy is preserved [20]. In order to fit these requirements, an approach based on geodesic front propagation and on Euclidian 3D distance map with respect to the background is used (Fig. 8). The propagation front is initiated at the open-end section of the trachea. Axis locations are defined by the distance map local maxima computed in the hyperplane associated to each propagation front. When several local maxima occur at
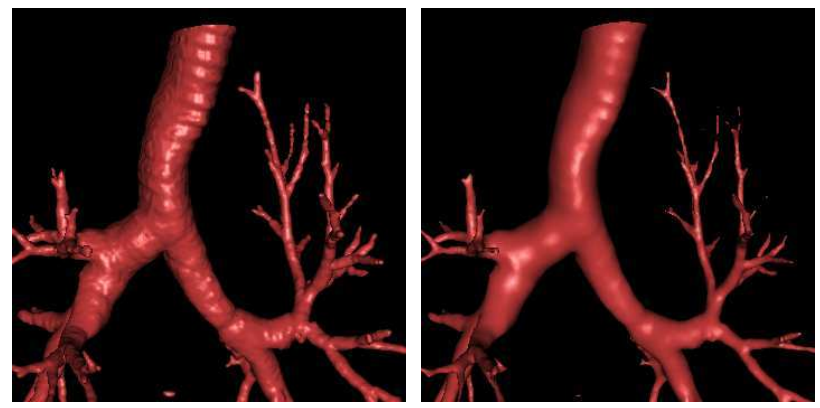

Figure 7: Marching cube-based surface meshing from 3D binary data with Gaussian pre-filtering. Left: small-size kernel, surface irregularities of the largest airways. Right: large-size kernel, geometry/topology errors of reconstruction of explored bronchi of smaller caliber.

the level of the current front, a bronchial subdivision is presumed. In order to avoid subdivision hierarchy errors due to noise or wall irregularities, a space partitioning is then performed, starting from the current axis location (Fig. 9). The space partitioning approach accurately detects subdivisions of quasi-tubular structures as well as their degree (bifurcation, trifurcation, ... ). First, at the current axis location point, the local maximal inscribed sphere is estimated, then its surface propagates toward lower values of the distance map. This gives birth to a cone-like shape inside each segment converging to the axis point of interest. When the cardinality of the set of cones is greater than 2 , the bronchus subdivision is validated. The correct geometry of the axis is reconstructed at the subdivision level by connecting each cone vertex to the current axis location along the maximal value path on the distance map. A new propagation front is defined at the level of each subtree and the axis is recursively built-up. Besides geometrical and topological properties, the axis determination includes at each point the information on the local bronchus caliber, derived from the 3D distance map. An average diameter can be then associated to each airway segment which is defined as the influence region of the corresponding axis branch. 


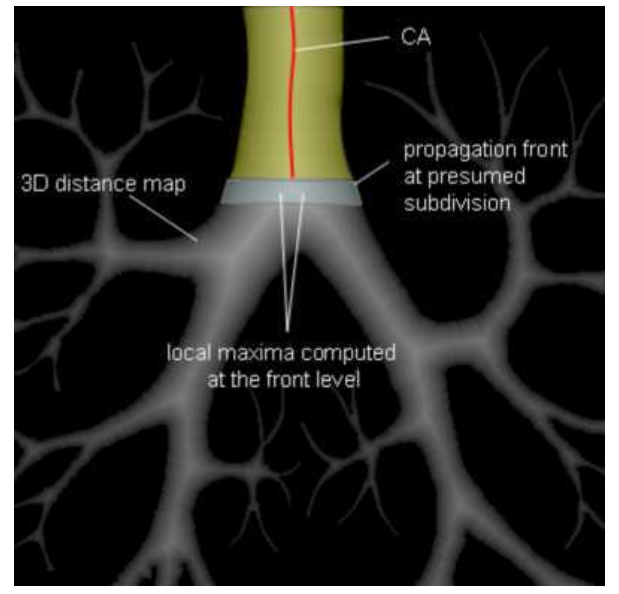

Figure 8: Principle of axis set computation based on geodesic front propagation and 3D distance map.
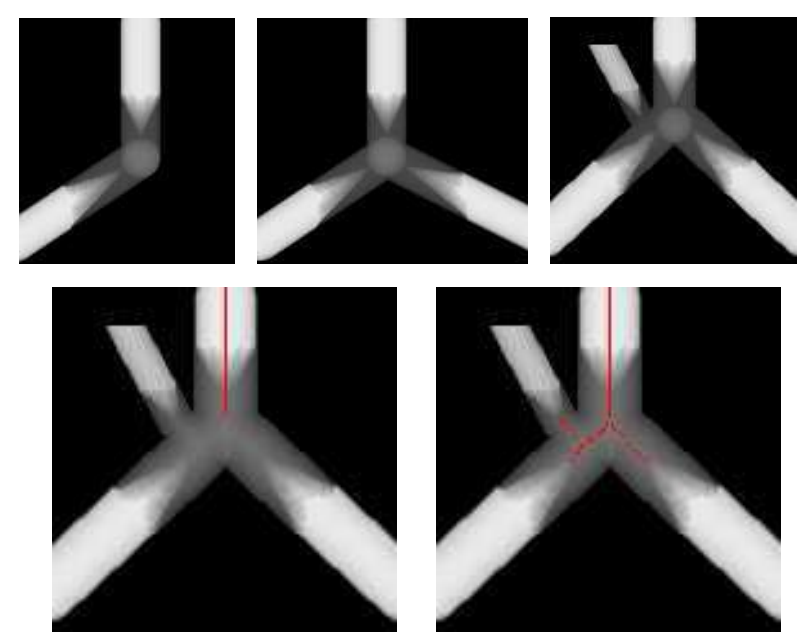

Figure 9: Space partitioning principle. Top: robustness with respect to the subdivision degree. Bottom: axis reconstruction at the subdivision level.

\section{Adaptive meshing}

Based on the average diameter information associated with each point of the segmented bronchial tree, the surface mesh is built as follows. The segmented binary data volume is first smoothed using an adaptive Gaussian kernel, of size and standard deviation varying with the average bronchus diameter. The objective is to achieve a constant caliber increasing ratio with respect to the binary structure, for all airway segments. By locally adjusting the isovalue parameter during the marching cube procedure, the mesh surface can be fitted on the real bronchus caliber, given by the segmentation. In practice, the same effect is obtained by using a constant isovalue (at the middle of the grayscale range) and by locally modifying the smoothed data values with respect to the isovalue and to the bronchus average diameter. Thereby, mesh topology errors (holes) are avoided and a fast implementation of the classic marching cubes algorithm can be used. The commercial software AMIRA serves for this purpose. For both smoothing and isovalue setup, the adaptation is fully-automated and uses pre-computed lookup tables. Fig. 10 shows a comparison between the mesh obtained with the adaptive and classic marching cubes procedures respectively. The adaptive approach provides a smooth mesh while preserving the original caliber of the trachea and the bronchi.
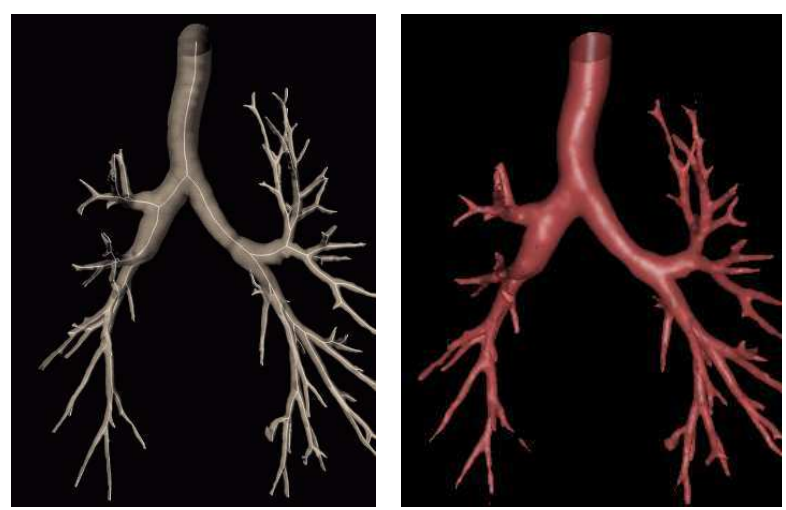

Figure 10: Surface model of the tracheobronchial tree with a generalized thickening of the bronchial sheaths, which is clearly observed in the right upper lobe. Left: adaptive technique. Right: classic marching cubes approach.

\section{Virtual bronchoscopy}

The 3D reconstruction of the tracheobronchial tree combined with the axis informations provide interactive tools for clinical investigation and treatment planning with a "virtual bronchoscopy" mode. Endoluminal navigation is performed along a trajectory which is automatically computed between two user-defined points on the axis network. The virtual camera is kept centered inside the set of explored airways providing an optimal view angle. The tracheobronchial tree displayed in Fig. 10 is used to illustrate the virtual bronchoscopy. The views obtained with the virtual bronchography mode correspond to a selected path from the top of the trachea, through the left main bronchus, the left upper lobe bronchus, and the apical segment of the latter (Fig. 11). 

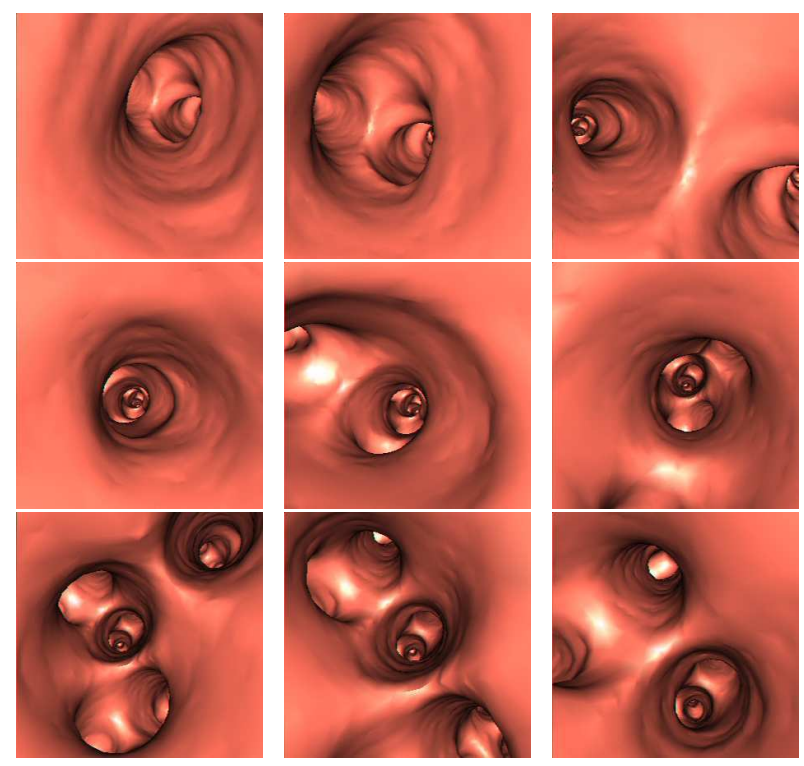

Figure 11: Virtual bronchoscopy views along a selected airway path, from the trachea to the left upper lobe apical bronchus, based on the axis data set of the model exhibited in Fig. 10.

\section{Computational mesh}

The surface meshes must match strong requirements related both to the accuracy of the surface approximation and to the element shape and size quality for the computations. The surface constructed from the very large set of noisy discrete informations must be simplified in order to get a suitable number of triangles to define the complex wall geometry of the $3 \mathrm{D}$ pipe network. Redundant elements are removed while preserving the accuracy of the geometric approximation of the underlying surface. The simplification procedure is based on the Hausdorff distance. This algorithm involves vertex deletion and edge flipping. The required smoothing is based on a bi-laplacian operator [24]. This stage yields to a geometric surface mesh that is a good approximation of the surface geometry and contains far less nodes than the initial reference mesh [7]. Surface smoothing and triangle-number optimisation (the triangulation is coarsened in the regions where the local curvatures are sufficiently large, whereas the main local curvatures are kept; the mesh is enriched in sharp regions with possible flow complex behaviour) is done using the different abilities of "YAMS" software [8].

The surface discretization obtained from the 3D reconstruction needs further treatment to be suitable for numerical simulations. The boundary conditions must be set at cross sections sufficiently far from the exploration volume in order to avoid pressure cross gradient and boundary-dependent flow disturbances, and to keep both upstream and downstream 3D effects of the pipe geometry [27].

Element shapes and sizes must be controlled as they usually impact the accuracy of the numerical results. Therefore, an anisotropic geometric metric map based on the local principal directions and radii of curvatures is constructed in the tangent planes related to the mesh vertices. This metric map prescribes element sizes proportional to the local curvature of the surface [9].

The conforming surface triangulation must be topologically accurate to generate a volumic mesh. The last step provides a tetrahedral mesh, using the fully automatic tetrahedral mesh generator "GHS3D" (TetMesh, distributed by SIMULOG) [10]. The numbers of points and elements for the computational meshes are given in Tab. 1.

\begin{tabular}{|c|c|c|c|c|}
\hline & M1a & M1b & M1c & M2 \\
\hline surface & 42692 & 83328 & 167492 & 162520 \\
\hline volume & 173788 & 521468 & 1077624 & 1340723 \\
\hline
\end{tabular}

Table 1: Element numbers of the surface discretization (triangles) and of the volumic mesh (tetrahedra) of the normal tracheobronchial tree (M1) and the pathological one (M2). Three M1 meshes have been used, from a coarse one (M1a) to a relatively fine one (M1c).

\section{Flow computations}

\section{Governing equations}

The air inhaled and exhaled during quiet breathing is supposed to be homogeneous, incompressible and Newtonian. The governing equations of an airway flow are derived from the mass and momentum conservations:

$$
\rho\left(\mathbf{u}_{t}+(\mathbf{u} \cdot \nabla) \mathbf{u}\right)=\mathbf{f}+\nabla \cdot \mathbf{C} ; \quad \nabla \cdot \mathbf{u}=0
$$

where $\mathbf{u}$ is the fluid velocity (subscript $t$ : time derivative), $\mathbf{f}=-\nabla \Phi$ the body force density ( $\Phi$ : potential from which body force per unit volume are derived), $\mathbf{C}=-p_{i} \mathbf{I}+\mathbf{T}$ the stress tensor, $(\mathbf{T}=2 \mu \mathbf{D}$ : extrastress tensor, $\mathbf{D}=\left(\nabla \mathbf{u}+\nabla \mathbf{u}^{T}\right) / 2$ : deformation rate tensor, I: identity tensor). With the given fluid properties, the Navier-Stokes equation becomes:

$$
\rho\left(\mathbf{u}_{t}+(\mathbf{u} \cdot \nabla) \mathbf{u}\right)=-\nabla p_{i}^{*}+\mu \Delta \mathbf{u} .
$$

where $p_{i}^{*}=p_{i}+\Phi$. The pressure notation is simplified in the following sections $\left(p_{i}^{*}=p\right)$.

The dimensionless governing equations

$$
S t \tilde{\mathbf{u}}_{\tilde{t}}+(\tilde{\mathbf{u}} \cdot \widetilde{\nabla}) \tilde{\mathbf{u}}-\operatorname{Re}^{-1} \widetilde{\Delta} \tilde{\mathbf{u}}+\widetilde{\nabla} \tilde{p}=0 ; \quad \tilde{\nabla} \cdot \tilde{\mathbf{u}}=0
$$


are obtained when dimensionless quantities are given by $\tilde{t}=t / T^{\star}, \tilde{x}=x / L^{\star}, \tilde{u}=u / U^{\star}, \tilde{p}=p /\left(\rho U^{\star 2}\right)$, and where $S t$ and $R e$ are the Strouhal and the Reynolds number respectively. With the unity scales usually taken (the dimensionless aerodynamics quantities have the same magnitude than the corresponding dimensional ones), the dimensionless governing equations become:

$$
\tilde{\mathbf{u}}_{\tilde{t}}+(\tilde{\mathbf{u}} \cdot \tilde{\nabla}) \tilde{\mathbf{u}}=-\widetilde{\nabla} \tilde{p}+\nu \widetilde{\Delta} \tilde{\mathbf{u}} ; \quad \tilde{\nabla} \cdot \tilde{\mathbf{u}}=0 .
$$

The boundary of the fluid domain is partitioned into a surface set: $\Gamma_{1}$, the cross section of the tracheal end, at which a Dirichlet boundary condition is applied, $\Gamma_{2}$, the cross sections of the bronchial ends, over which normal constraint is equal to zero, and $\Gamma_{3}$, the airway wall, which is assumed to be rigid. The classical no-slip condition is applied to the airway wall. A timedependent uniform injection velocity is prescribed at the inlet which provides a zero-mean sinusoidal flow.

During quiet breathing (tidal volume $V_{T}=500 \mathrm{ml}$ and respiratory frequency $f=0.25 \mathrm{~Hz}$ ), the Mach number $M a<0,2$ and the Helmholtz number

$H e=\omega L / c \leq 0.0006$ ( $L$ : airway length, $c$ : wave speed, $\omega$ : pulsation). The inhaled air can thus be considered as incompressible. It is heated, being at the body temperature $(T=37 C)$. Moreover, it is saturated with water vapor. Consequently the physical properties of the air in the respiratory tract are the followings: the air density $\rho=19.0410^{-6} \mathrm{~kg} \cdot \mathrm{m}^{-3}$, the dynamic viscosity $\mu=1.068 \mathrm{Pl}$ and the kinematic viscosity $\nu=17.810^{-6} \mathrm{~m}^{2} . \mathrm{s}^{-1}$.

The values of the peak tracheal Reynolds numbers $\widehat{R e}$ based on the peak cross-sectional average velocity and on the tracheal radius at the end cross section are equal to 700,750 and 850 . The Stokes numbers Sto, based on the radius at the cross section of the tracheal end and the peak Strouhal number $\widehat{S t}$ are equal to 4.2 or 4.7 and 0.025 respectively.

\section{Numerical simulations}

The numerical technique to solve the classical fluid mechanics equations for incompressible fluids is the finite element method. It is based on a variational formulation of the Navier-Stokes equations on a domain $\Omega$ :

$$
\begin{array}{r}
\mathcal{B}(\mathbf{u}, \mathbf{v})+\mathcal{T}(\mathbf{u} ; \mathbf{u}, \mathbf{v})+\mathcal{B}^{\prime}(\mathbf{v}, p)=<l, \mathbf{v}>, \\
\forall \mathbf{v} \in V \subset H^{1}(\Omega)^{3}, \\
\mathcal{B}^{\prime}(\mathbf{u}, q)=0, \forall q \in Q \subset L^{2}(\Omega)
\end{array}
$$

where $\mathcal{B}$ et $\mathcal{B}^{\prime}$ are bilinear forms, $\mathcal{T}$ a trilinear form, $\langle l, \mathbf{v}\rangle$ the dual product (the quantity $l$ takes into account the nonhomogeneous velocity and possible pressure boundary conditions as well as the possible forcing term f), $H^{1}(\Omega)$ and $L^{2}(\Omega)$ the Sobolev space of order 1 defined on $\Omega$ for vector-valued functions and the space of functions that are square integrable in the Lesbegue sense with respect to $\Omega$ respectively.

The computational method is suitable to unsteady flow, the time being used as an iterative parameter of the solution. The finite element type is $P_{1}-P_{1}$ bubble element [1]. The pressure $\tilde{p}$ is defined at the four vertices of the tetrahedron $\left(P_{1}\right.$ element) and the velocity $\tilde{\mathbf{u}}$ at both the vertices and the barycenter ( $P_{1}$ bubble element). The order of the method is $\mathcal{O}\left(\xi^{2}\right)$ for $\tilde{\mathbf{u}}$ and $\mathcal{O}(\xi)$ for $\tilde{p}_{i}$ in the $L^{2}$ norm, $\xi$ being the characteristic size of the tetrahedron. The convective term is approximated by the method of characteristics [22]. The solution is obtained via a generalized Uzawa-preconditioned-conjugate gradient method [11]. The initial condition is given by a Stokes problem with the same boundary condition as the unsteady one (period of $1 s$ ).

\section{RESULTS}

The time variations of the flow rate and the pressure are plotted in Fig. 12. A strong phase difference is observed between the traces of the flow rate and of the pressure in the model M2 of the diseased tracheobronchial tree. Moreover, a non-symmetrical cyclic variation of the pressure occurs. The duration of the sum of the deceleration phase of expiration and of the acceleration phase of inspiration is twice the duration of the sum of the decelerating phase of inspiration and of the accelerating phase of expiration.

Six phases have been selected to depict the numerical results: mid acceleration phase, peak, and mid deceleration phase of inspiration, mid acceleration phase, peak, and mid deceleration phase of expiration. The streamlines in M1 are displayed at peak inspiration and expiration in Fig. 13. Due to the cumulative effects of bronchial curvature, the streamlines exhibit a larger helical motion in the stem bronchi during expiration than during inspiration, the trachea being straighter than the bronchi. The streamlines show that the stenosis disturbs the flow, the right part of M2 being poorly irrigated (Fig. 14). At the entrance segment of the left main bronchus, the flow is not only governed by the geometrical curvature, but also by a remote dynamical bending effect generated by the stenosis of the right main bronchus.

The paths of particles injected at the tracheal end have been drawn. A particle bolus is observed during the acceleration phase of the inspiration (Fig. 15). The particle locations are dispersed at peak flow. The particle scattering increases during the deceleration phase 

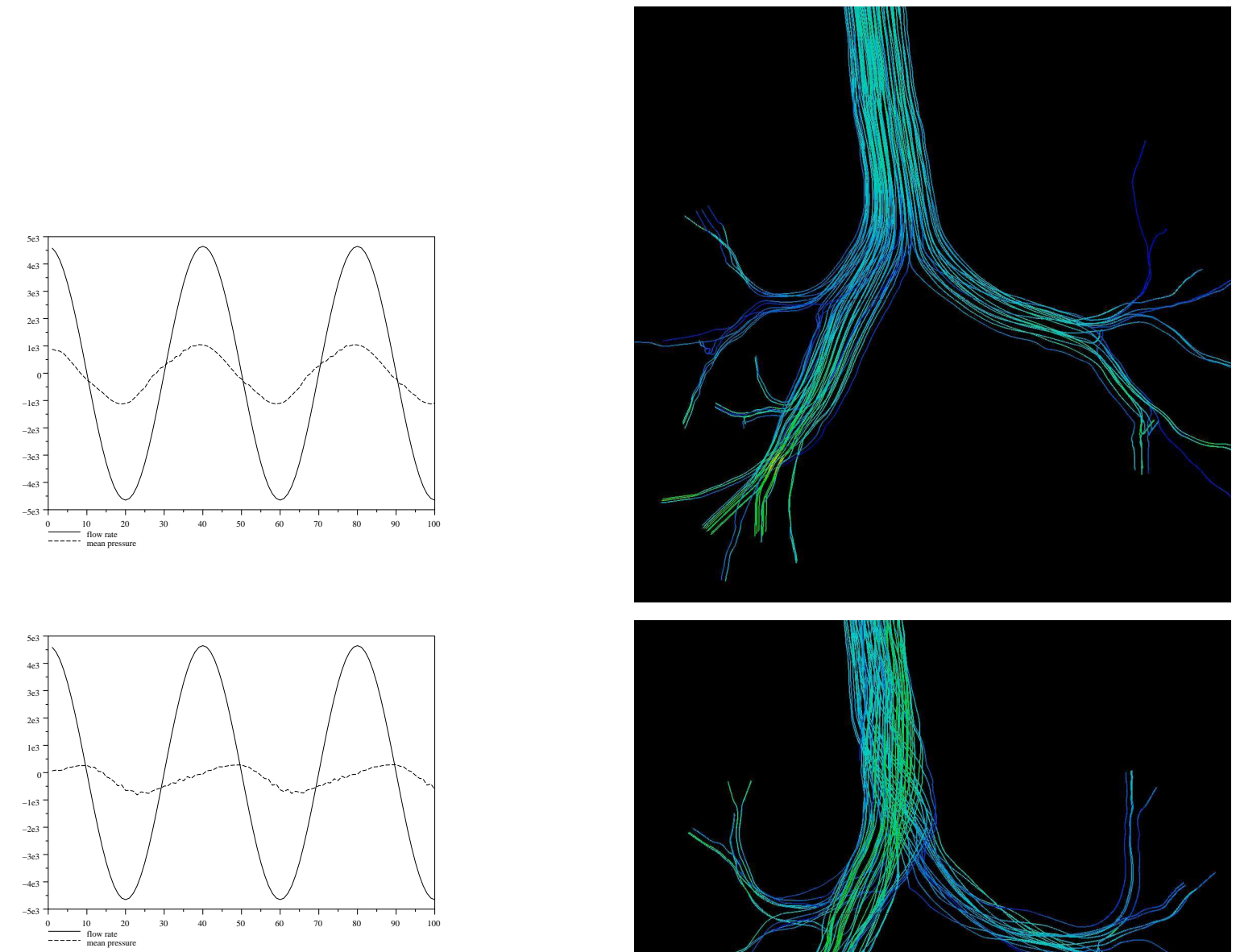

Figure 12: Temporal variation of the flow rate (continuous line) and the cross sectional average pressure (discontinuous line) at the tracheal end. Model M1 (left) and M2 (right).

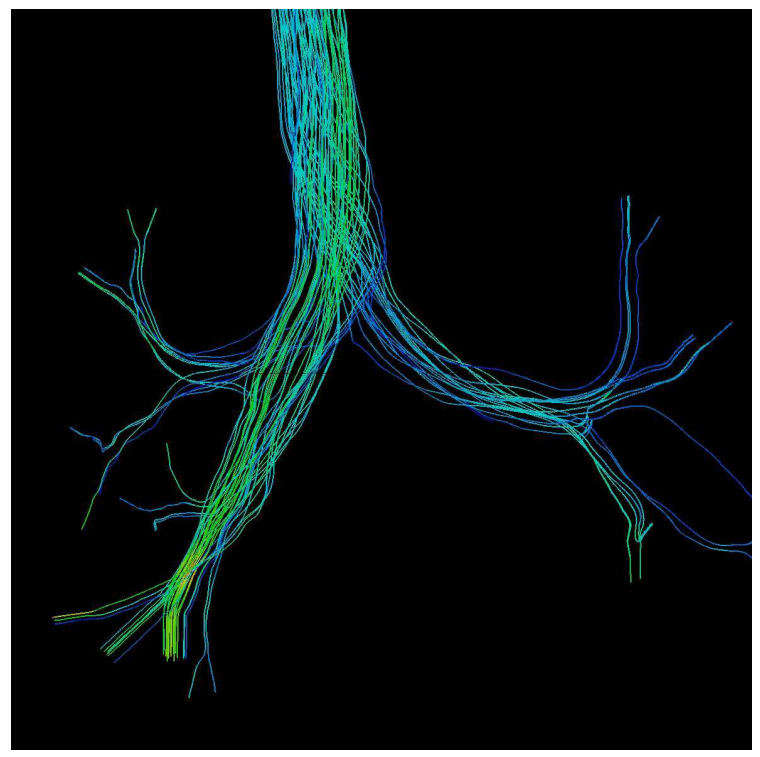

Figure 13: Streamlines in model M1 at peak inspiration (top) and expiration (bottom). 


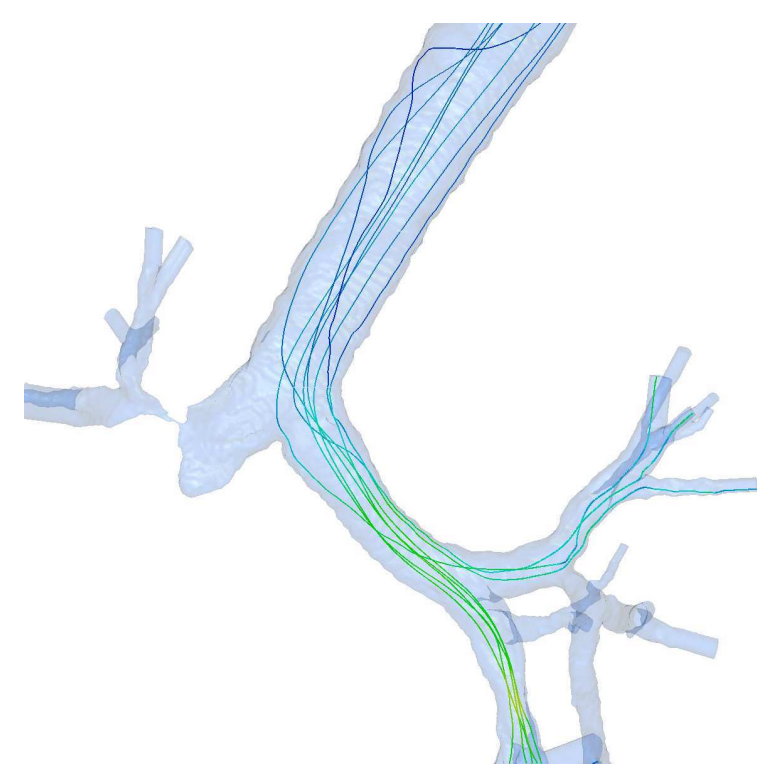

Figure 14: Streamlines in zoomed model M2 during the decelerating phase of inspiration.

of the inspiration, the particle reaching the bronchi. The particles, which travel backwards, toward the trachea, during the subsequent expiration, remain dispersed (Fig. 16).

The flow distribution can be calculated for the five main compartments, or lobes, of the two lungs, the corresponding bronchi being put together. Pulmonary lobe ventilation of model M1, consequently, is associated with superior (SRL), middle (MRL) and inferior (IRL) right and with superior (SLL) and inferior (ILL) left lobes. The flow distribution is estimated, rather than properly quantified, due to the crude boundary conditions used in the present numerical simulations, since the impedance of the small bronchi is unknown. The relative flow contribution, given at the selected phases of the respiratory cycle, of the right bronchus set (Tab. 2) with respect to the one of the left tree part (Tab. 3) is higher during the expiration than during the inspiration. Such results can be due to the chosen boundary conditions, the bronchial ends being free and the flow set up at the tracheal end. More air is exhaled from the right bronchial network because of a greater number of bronchial ends with respect to the left tree part. For a given lobe, the relative difference between the maximum and the minimum of its flow contribution during the respiratory cycle can reach $25 \%$. Similar trends have been obtained with different sets of values of flow governing parameters.
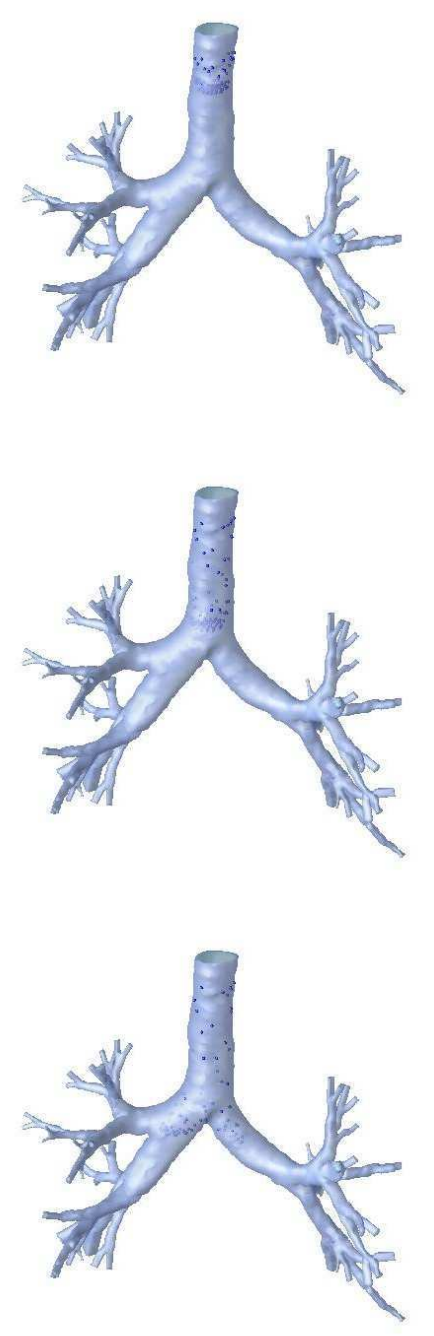

Figure 15: Particle paths during inspiration (mid acceleration phase, peak, and mid deceleration phase) from top to bottom. 

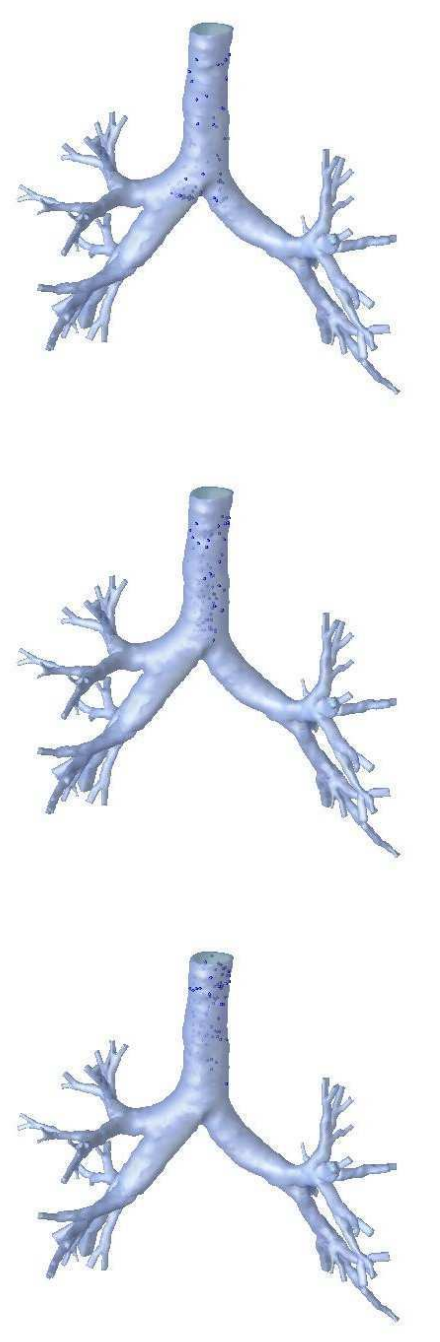

Figure 16: Particle paths during expiration (mid acceleration phase, peak, and mid deceleration phase) from top to bottom.
Table 2: Between-lobe flow distribution in the right lobes of M1 (R: right, S: superior, M: middle, I: inferior) at selected phases of the respiratory cycle (MAI: mid acceleration phase of inspiration, PI: peak inspiration, MDI: mid deceleration phase of inspiration, MAE: mid acceleration phase of expiration, PE: peak expiration, MDE: mid deceleration phase of expiration, $q_{R}$ : flow in the right part of the tracheobronchial tree, $q_{t}$ : tracheal flow rate).

\begin{tabular}{|c|c|c|c|c|}
\hline phase & RSL & RML & RIL & $q_{R} / q_{t}$ \\
\hline MAI & 0.15 & 0.08 & 0.37 & 0.60 \\
\hline PI & 0.13 & 0.07 & 0.40 & 0.60 \\
\hline MDI & 0.14 & 0.08 & 0.38 & 0.60 \\
\hline MAE & 0.17 & 0.08 & 0.38 & 0.64 \\
\hline PE & 0.17 & 0.09 & 0.39 & 0.66 \\
\hline MDE & 0.16 & 0.09 & 0.42 & 0.67 \\
\hline
\end{tabular}

Table 3: Between-lobe and between-lung flow distribution in the left lobes of M1 (L: left, S: superior, I: inferior) at selected phases of the respiratory cycle (MAI: mid acceleration phase of inspiration, PI: peak inspiration, MDI: mid deceleration phase of inspiration, MAE: mid acceleration phase of expiration, PE: peak expiration, MDE: mid deceleration phase of expiration $q_{L}$ : flow in the left parts of the tracheobronchial tree, $q_{t}$ : tracheal flow rate).

\begin{tabular}{|c|c|c|c|}
\hline phase & LSL & LIL & $q_{L} / q_{t}$ \\
\hline MAI & 0.16 & 0.24 & 0.40 \\
\hline PI & 0.15 & 0.25 & 0.40 \\
\hline MDI & 0.16 & 0.24 & 0.40 \\
\hline MAE & 0.15 & 0.21 & 0.36 \\
\hline PE & 0.14 & 0.20 & 0.34 \\
\hline MDE & 0.12 & 0.21 & 0.33 \\
\hline
\end{tabular}




\section{DISCUSSION}

Numerical mesh Airway junctions give birth to bronchi of smaller size with given branching angles. The bronchus branches off not only with an angle in the plane of the junction (a short duct length over which the trunk axis can be considered planar), but also outside this plane. Therefore, the tracheobronchial tree must be carefully reconstructed. If the thorax has been scanned from the neck to the abdomen, the longitudinal axis of the reconstructed model must be oriented in the same direction, keeping the direct reference trihedron. Otherwise, a bronchus going to the ventral side of the body can take a dorsal direction.

Geometrical features at small length scale of the airway wall can affect the flow. The walls of the trachea and of the main bronchi are made of cartilaginous U-shaped rings. The cartilage makes closed rings in bronchi of higher-order generations. The cartilaginous rings thicken the wall. The wall thickness of the intercartilaginous membranes is equal to about $80 \%$ of the wall thickness of the cartilaginous segment. Such a width difference (about $0.6 \mathrm{~mm}$ ) is equal to the transverse spatial resolution of the MSSCT. The height of the intercartilaginous membranes represents $30-45 \%$ of the height of the cartilage [2]. Although the existence of these reinforcing bands can be guessed by examination of the airway surface, they were not accurately reconstructed. Such a drawback is not very important as long as aerosols are not involved.

The volumic mesh must preserve a thin near-wall layer throughout the computational model. The greater the width of the peripheral (near-wall) layer of mesh cells of the cross section of the tracheal end, the higher the error on the injected flow rate using an injection velocity condition (Dirichlet condition).

The discretised M1 model has been investigated with different element densities (Tab. 1). Comparisons of interpolated values of aerodynamical quantities in arbitrary chosen points of the volumic mesh have been made. The relative differences are given in Tab. 4 , taking the velocity magnitude $\|u\|_{M 1 c}$ of the high-density mesh as a reference. The velocity profiles at selected cross-sections of the tracheobronchial model are almost superimposed for the three mesh densities (Fig. 17). Moreover, the bulk flow properties remains unchanged.

Air physical properties Although the air physical properties do not strongly affect the pressure and the velocity in a steady flow, higher differences are observed in an oscillatory flow, especially during the respiratory cycle phases close to flow reversal (Tab. 5).
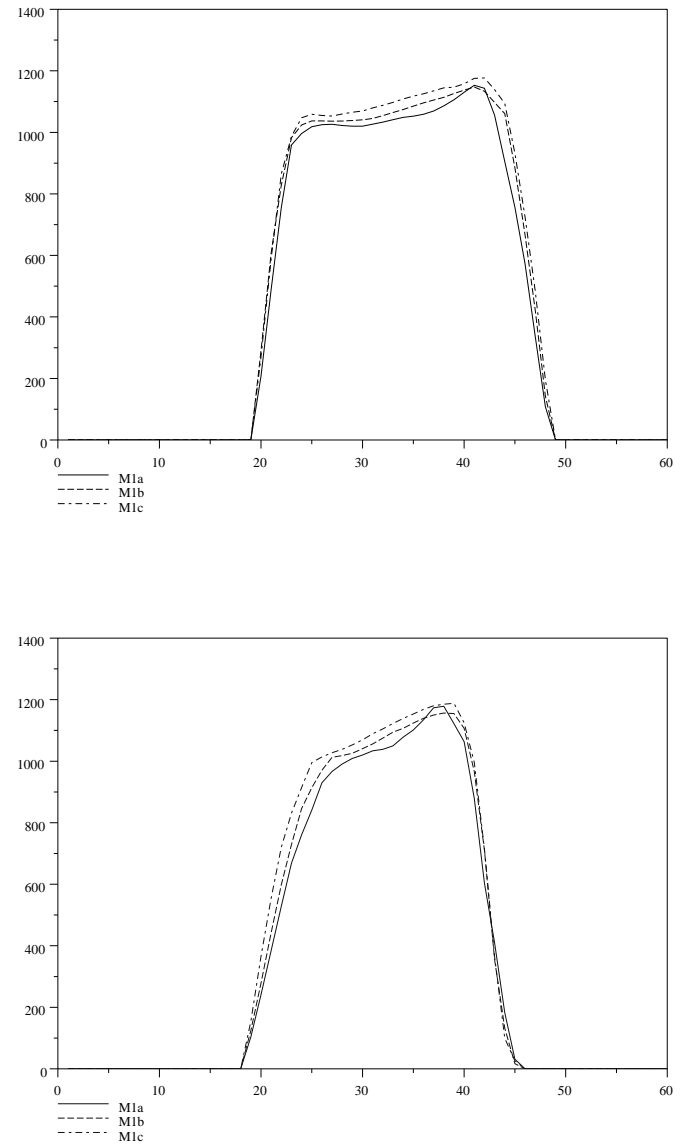

Figure 17: Velocity profiles in two orthogonal planes of a tracheal section immediately upstream from the transition zone computed using the three M1 meshes, M1a (continuhous line), M1b (dashed line) and M1c (dashed-dotted line). 
Table 4: Flow velocity $\left(\|u\|_{M 1_{k}}-\|u\|_{M 1 c}\right) /\|u\|_{M 1 c}$ and pressure $\left(p_{M 1_{k}}-p_{M 1 c}\right) / p_{M 1 c}$ between-mesh relative differences in two selected points $(k=a, b)$. The two points are located either in the trachea, near the tracheal bifurcation $P 1:(196.5,160.5,145.5)$ or in the main left bronchus $P 2$ : $(218.8,165.1,123.4)$.

\begin{tabular}{|l|c|c|c|}
\hline & & M1a & M1b \\
\hline P1 & velocity & 0.07 & 0.03 \\
& pressure & 0.01 & 0.03 \\
\hline P2 & velocity & 0.05 & 0.03 \\
& pressure & 0.03 & 0.03 \\
\hline
\end{tabular}

Table 5: Effect of air physical properties on steady flow and at inspiration peak (PI), expiration peak (PE) and at instants close to flow reversal, from inspiration to expiration (I/E) and from expiration to inspiration (E/I). Relative difference in pressure and velocity between ambient air $\left(\nu=14.6 \mathrm{~mm}^{2} / \mathrm{s}\right)$ and lung air $\left(\nu=17.8 \mathrm{~mm}^{2} / \mathrm{s}\right)$.

\begin{tabular}{|c|c|c|}
\hline phase & pressure & flow \\
\hline \multicolumn{3}{|c|}{ steady flow } \\
\hline & 0.04 & 0.10 \\
\hline \multicolumn{3}{|c|}{ oscillatory flow } \\
\hline $\mathrm{I} / \mathrm{E}$ & 0.58 & 0.68 \\
\hline $\mathrm{PE}$ & 0.20 & 0.21 \\
\hline $\mathrm{E} / \mathrm{I}$ & 0.78 & 0.50 \\
\hline PI & 0.22 & 0.13 \\
\hline
\end{tabular}

Boundary conditions The types of boundary conditions set at the ends of both the trachea and the bronchi affect the flow inside the computational domain, especially the flow distribution. The upper airways mainly influence the velocity profiles at the tracheal inlet during the inspiration. The flexible constriction of the larynx induced a tracheal jet. Assuming a symmetrical narrowing of the larynx, whatever its deformation during the respiratory cycle, and only a slight curvature of the trachea, the approximately axisymmetrical tracheal jet do not strongly affect the flow distribution between the stem bronchi. The major factor is then the impedance of the various bronchial paths, distal to the computational models. The branchings, indeed, continue down to the terminal bronchioles (the smallest airways without alveoli), then to the respiratory bronchioles, with more and more side alveoli as the generation index increases. The lung acinus is the parenchyma region, composed of the respiratory bronchioles and the alveolar ducts.

Downstream from the computational domain composed of a tree with G0 to G6/G7, the bronchi can be consid- ered either as a unique element from G7/G8 to the alveolar level, which comprises a set of in parallel compartments for the considered set of lung territories, or made of three compartments in series. In the first modelling kind, the bronchi can be represented using lumped parameter models. In the second type, the small bronchi are modelled by a set of short ducts and junctions, the bronchioles by a fractal tree, and the airways within the pulmonary acini by a deformable reservoir.

The flow in the compartment G8-G13 of the bronchial tree can be approximately solved using the reduced basis element $[16,17]$, the architecture of this hierarchic system being not completely repetitive. This compartment can be defined as the juxtaposition of generic elements to which reduced basis functions can be associated. These elements can be obtained from the deformation of reference pipes and junctions. The whole mathematical system is reconstructed using Lagrange multipliers. Although the bronchial tree cannot be accurately described by a fractal object, because the nature deals neither with regular configurations nor with infinite iterations, the fractal model can be used as an approximative representation of a part of the bronchial tree (G14-G18), assumed to be characterized by a regular dichotomy. The fractal dimension is then defined by $D=-\ln (2) / \ln (s), s<1$ is the scaling factor between the generations $G_{i}$ and $G_{i+1}$ $\left(L_{i+1}=L_{i} / s, d_{i+1}=d_{i} / s\right)$. In the Weibel model, $s \sim 0.82$ (the bronchial tree fills a finite space). Therefore, $D \sim 3.49$. The repetitive architecture of the compartment G14-G18 of the bronchial tree prompts to use the homogeneization theory associated with a scaling factor (fractal homogeneization) [18]. The relative spatial periodicity of the lung acini also leads to the homogeneization theory. However, this distal compartment, at least, deforms during breathing, inflating during inhalation and deflating during exhalation. The unsteady geometry is associated with volume variations. Further mathematical and numerical analysis remains to be carried out on these topics, examples of possible computational models of small intraparenchymal airways.

The design of the present bronchial networks does not end to a homogeneous generation level (G5 or G6 mainly). No rules can indeed be defined to set up the model endings because of the heterogeneous dimensions of the bronchi which belong to a given generation. The geometrical properties of a given bronchus of the G5 family can be closer to those of a G6 airway. Different boundary conditions could be set at the various bronchial ends of the model of the tracheobronchial tree, taking into account the height of the bronchial ends within the thorax. The hydrostatic pressure, indeed, decreases with height at a rate of $\sim 0.1 \mathrm{~Pa} / \mathrm{cm}$ in the airways. Another work stage then deals with the implementa- 
tion of a variational formulation suitable for pressure boundary conditions [4].

Because in the present work simple boundary conditions have been used at the model ends, the velocity field has not been investigated in details. Most of the results focus on bulk flow features and on estimations of the flow distribution among the set of bronchi. The features of possible flow separations depend not only on the local geometry but also on flow distribution associated with the boundary conditions [12]. Explorations of flow separations have not been performed, although they are involved in aerosol deposition. The secondary motions, a virtual fluid displacement corresponding to the projection of the local velocity field in the plane of any cross section, have not been investigated. The major fluid motion is helical as depicted by the streamlines.

Although reduced-dimension models, like zero- and onedimensional models, can give the flow distribution among the bronchi, they cannot suit 3D flows. Pathological states, with localized lesions, emphasize this feature. The present modelling strategy is required for computer-aided medicine.

Numerical method Two finite element techniques have been used, (i) a home-made software NSI3 based on P1-bubble P1 tetrahedra and (ii) the commercial Fidap package based on P1- P1 tetrahedra associated with SUPG and PSPG formulations. The meshes are not strictly identical. The results, given in Tab. 6 , show that the relative differences in quantity maxima can reach values up to $20 \%$. However, the flow distribution is not significantly different.

Table 6: Between fluid-solver comparison (Fidap vs. home-made solver NSI3). Steady flow corresponding to peak inspiration.

\begin{tabular}{|l|c|c|}
\hline & Fidap & NSI3 \\
\hline elements & 986982 & 1077624 \\
\hline $\begin{array}{l}p_{\max } \\
(P a)\end{array}$ & 2.73 & 3.40 \\
\hline $\begin{array}{l}\|u\|_{\max } \\
(\mathrm{m} / \mathrm{s})\end{array}$ & 2.18 & 2.35 \\
\hline$q_{R} / q_{t}$ & 55.3 & 57.0 \\
$\frac{q_{R S L}}{q_{R I L+R M L}}$ & 1.2 & 1.1 \\
$\frac{q_{L S L}}{q_{L I L}}$ & 2.4 & 2.6 \\
\hline
\end{tabular}

Flow stability Like steady flows, time-varying flows are stable if any disturbance is spontaneously eliminated, due to the dissipative action of the viscous forces, a major source of instabilities being vortex production. Otherwise, self-modulated perturbations can lead to evolving small scale structures. Periodic flows are transiently stable if a disturbance grow during a part of the cycle and decays. Periodic flows are unstable when the disturbance grows during each period.

A critical dimensionless parameter is a ratio of destabilizing to stabilizing forces. When the threshold is exceeded at any point of the fluid domain, the flow destabilization is not counterbalanced by restoring forces and the disturbances grow. The critical Reynolds number $R e_{c r i t}$, at which transition from laminar to turbulent pattern occurs, is suitable for steady developed flows in straight rigid pipes. This threshold can be pushed upward to $\sim 10^{5}$, when extreme care is done to reduce disturbances [5]. Pipe curvature damps turbulences in steady flows [25]. The higher the curvature ratio, the greater $R e_{\text {crit }}$. However, when disturbances are small, $R e_{\text {crit }}$ in bends can be lower than in straight pipes. $R e_{\text {crit }}$ appears thus to depend on the perturbation amplitude in steady flow. Distensible straight pipes damp existing turbulence in steady flow, the turbulence intensity being lower than in rigid pipes [23]. Destabilization/stabilization of a time-dependent flow also depends on the frequency and on the magnitude of the flow. A critical Strouhal number, based on the unsteady boundary layer thickness, has been used for unsteady motions. The Stokes number Sto, which ressembles to $R e$, the convective inertia being replaced by the unsteady inertia in the force ratio, is an involved frequency parameter. The higher the Stokes number Sto, the lower the time available for disturbance growth. Demarcation of the flow patterns is commonly made on Sto-Re diagrams.

The most common idealized airway model has been proposed by Weibel [28] (Tab. 7). It assumes a regular symmetrical dichotomy with even flow distribution among the branches at each junctions. Let $q_{i}=q_{0} / 2^{i}$ be the flow rate in any bronchus of the generation $i$. When the flow waveform is supposed to be sinusoidal (equal inspiration and expiration durations), the respiratory volume is $V=1 / 4 \omega V_{T} \sin (\omega t)$ and $q=1 / 4 \omega^{2} V_{T} \cos (\omega t)$. In such an idealized model, using the above-mentioned assumptions, the values of the flow governing parameters can then be calculated (Tab. 8).

Several features affect the flow stability, the vessel curvature with respect to the disturbance amplitude, the wall deformability, and the flow unsteadiness. Transitional threshold (critical values) of the flow dimensionless parameters can only be used in similar configura- 
Table 7: Lengths $L(\mathrm{~mm})$, hydraulic radii $R_{h}(\mathrm{~mm})$, aspect ratios $L / d_{h}\left(d_{h}\right.$ : diameter), and cross sectional areas $A\left(\mathrm{~mm}^{2}\right)$ of the first generations (G) of the symmetrical dichotomy model of the tracheobronchial tree.

\begin{tabular}{|c|c|c|c|c|}
\hline $\mathrm{G}$ & $L$ & $R_{h}$ & $L / d_{h}$ & $A$ \\
\hline 0 & 120 & 9 & 6.7 & 254 \\
\hline 1 & 47 & 6 & 3.9 & 113 \\
\hline 2 & 19 & 4 & 2.4 & 50 \\
\hline 3 & 7.5 & 2.8 & 1.4 & 25 \\
\hline 4 & 12 & 2.2 & 2.7 & 16 \\
\hline 5 & 10 & 1.7 & 2.9 & 10 \\
\hline 6 & 9 & 1.4 & 3.2 & 6 \\
\hline
\end{tabular}

Table 8: Stokes Sto, Reynolds $\widehat{R e}$ and Strouhal $\widehat{S t}$ numbers based on the radius, or on the unsteady Stokes boundary layer thickness $\delta$ and the peak cross-sectional average velocity during rest breathing.

\begin{tabular}{|l|c|c|c|c|}
\hline $\mathrm{G}$ & Sto & $\widehat{R e}$ & $\widehat{R e}(\delta)$ & $\widehat{S t}$ \\
\hline 0 & 2.7 & 612 & 232 & 0.011 \\
\hline 1 & 1.8 & 459 & 261 & 0.007 \\
\hline 2 & 1.2 & 344 & 293 & 0.004 \\
\hline 3 & 0.8 & 246 & 300 & 0.003 \\
\hline 4 & 0.7 & 153 & 232 & 0.003 \\
\hline 5 & 0.6 & 98 & 190 & 0.003 \\
\hline 6 & 0.4 & 61 & 150 & 0.003 \\
\hline
\end{tabular}

tions of the flow domains. These values in the respiratory tract have not yet been determined. However, the values of the flow governing parameters in our numerical experiments are relatively small. Laminar flow in blood vessel corresponds to a weak assumption.

Time reproducibility The reproducibility of the aerodynamical quantities over successive periods varying with a zero-mean sinusoidal motion and the effect of computation initialization have been studied. The reproducibility of aerodynamical quantities has first been checked in selected planes of an image-based model of the respiratory duct, composed of the lower pharynx, the larynx and the trachea (a single pipe). The flowrate traces in multiple cross sections during successive respiratory cycles are wholly superimposed. Mean values are reproducible from the first period in different planes of the airway segment (Fig. 18). Relative difference between maximal values of the pressure and of the velocity at successive peak expiration and inspiration are given in Tab. 9. The cycle-to-cycle variations in pressure and in velocity are smaller at peak inspiration than at peak expiration.
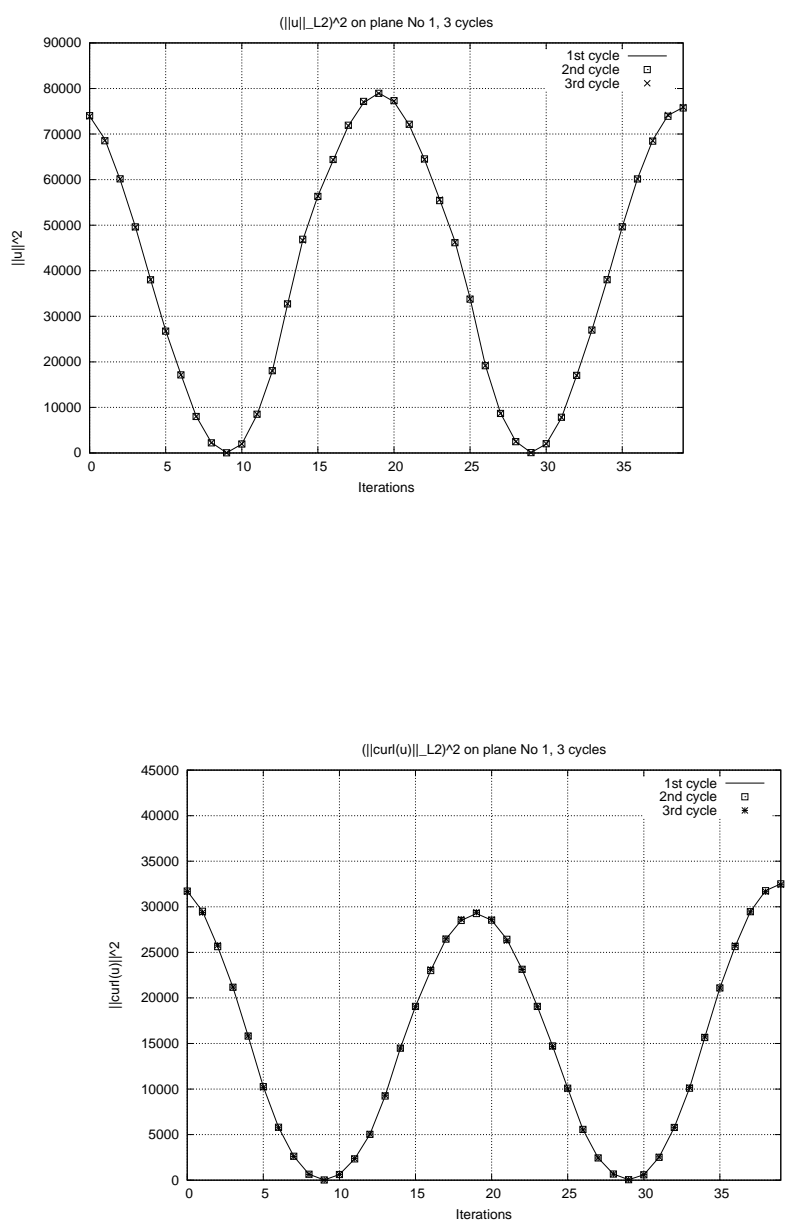

Figure 18: $L^{2}$ norm of the section averaged value of both the velocity and the velocity curl in selected planes during the three successive first periods. 
Table 9: Relative difference between maximal values of the pressure and of the velocity at peak expiration and inspiration between two successive cycles $i$ and $i+1$.

\begin{tabular}{|l|c|c|c|c|}
\hline & \multicolumn{2}{|c|}{ PE } & \multicolumn{2}{c|}{ PI } \\
\hline cycles & $p$ & $u$ & $p$ & $u$ \\
\hline $1-2$ & 0.03 & 0.17 & & \\
$2-3$ & 0.01 & 0.14 & 0.002 & 0.012 \\
$3-4$ & 0.01 & 0.05 & 0.002 & 0.011 \\
$4-5$ & 0.01 & 0.09 & 0.002 & 0.009 \\
$5-6$ & 0.01 & 0.08 & 0.002 & 0.010 \\
$6-7$ & 0.01 & 0.09 & 0.002 & 0.010 \\
$7-8$ & 0.01 & 0.10 & 0.003 & 0.012 \\
$8-9$ & 0.01 & 0.09 & 0.002 & 0.017 \\
$9-10$ & 0.03 & 0.19 & 0.002 & 0.020 \\
$10-11$ & 0.02 & 0.16 & 0.007 & 0.016 \\
$11-12$ & 0.02 & 0.14 & 0.006 & 0.010 \\
$12-13$ & 0.01 & 0.08 & 0.002 & 0.011 \\
$13-14$ & 0.01 & 0.12 & 0.002 & 0.008 \\
$14-15$ & 0.01 & 0.12 & 0.002 & 0.012 \\
$15-16$ & 0.02 & 0.12 & 0.001 & 0.012 \\
$16-17$ & 0.03 & 0.15 & 0.003 & 0.009 \\
$17-18$ & 0.01 & 0.06 & 0.003 & 0.009 \\
\hline
\end{tabular}

Airway deformability The airway wall, a composite heterogeneous material, is either wholly soft, like the pharynx or reinforced by cartilage, such as the first generations of the tracheobronchial tree. Any segment of the respiratory tract is deformable, experiencing distension and collapse [26]. Like every biological tissue, the airway wall is viscoelastic.

The images are acquired during breathholding. The possible variation in dimensions of the airway luminae remains unknown; they are supposed to be small during rest breathing, at most comparable to the spatial resolution of the available imaging techniques. Moreover, the rheology of the airway wall is not well developed, especially in vivo. The material constants associated with the constitutive laws of the bronchi have not been measured. The wall of the intrathoracic explored airways is, thereby, assumed to be rigid.

\section{CONCLUSION}

The present work is performed in the context of computer-aided diagnosis for lung diseases. Computer tools are developed, in this first stage, in order (i) to provide the best possible visualisation of the diseased region and its surroundings, as well as a navigation tool for endoluminal examination, and (ii) to give the flow features and the flow distribution among the lung territories. Thereby, the geometrical modelling is crucial for both objectives. Multiscale models must be devel- oped for the second goal, in order to take into account the presence of the airway compartments located at both ends of the tracheobronchial tree, i.e. the set of the upper airways at the first end, the smallest bronchi and the pulmonary acini at the other ends, rather than crude, but usual, boundary conditions.

Acknowledgements Support for this work was provided by the french ministry of research in the framework of project "R-MOD" of the Réseau National Technologies pour la Santé RNTS 2001, in association with Air Liquide company in particular. The authors thanks P. Grenier who provides the CT images, L. Baffico and P. Métier for the earlier set of computations using an image-based domain with some bronchi reconstructed frontside back, and R. Fodil who helps in discretizing the reconstructed surface, using AMIRA software.

\section{References}

[1] Arnold, D.N., Brezzi, F., Fortin, M. (1984) A stable finite element for the Stokes equations, Calcolo, 21, 337-344.

[2] Begis, D., Delpuech, C., Le Tallec, P., Loth, L, Thiriet, M, Vidrascu, M. (1988) A finite element model of tracheal collapse, J. Appl. Physiol., 64, 1359-1368.

[3] Cebral, J.R., Summers, R.M. (2004) Tracheal and central bronchial aerodynamics using virtual bronchoscopy and computational fluid dynamics, IEEE Trans Med Imaging, 23(8), 1021-1033.

[4] Conca, C., Pares, C., Pironneau, O., Thiriet M. (1995) A computational model of Navier-Stokes equations with imposed pressure and velocity fluxes. Int. J. Numer. Methods in Fluids, 20, 267287.

[5] Draad, A.A., Kuiken, G.D.C., Nieuwstadt, F.T.M. (1998) Laminar-turbulent transition in pipe flow for Newtonian and non-Newtonian fluids, J. Fluid Mech., 377, 267-312.

[6] Fetita, C., Prêteux, F., Beigelman-Aubry, C., Grenier, P. (2004) Pulmonary airways: 3D reconstruction from multi-slice CT and clinical investigation, IEEE Transactions on Medical Imaging, 23(11), 1353-1364.

[7] Frey, P.J., Borouchaki, H. (1998) Geometric surface mesh optimization, Computing and Visualization in Science, 1, 113-121. 
[8] Frey, P.J. (2001) A fully automatic adaptive isotropic surface remeshing procedure, INRIA Research Report, 0252.

[9] Frey, P.J., Borouchaki, H. (2003) Surface meshing using a geometric error estimate, Int. J. Numer. Methods Engng., 58, 227-245.

[10] George, P.L. (1997) Improvement on Delaunay based 3D automatic mesh generator, Finite Elements in Analysis and Design, 25(3-4), 297-317.

[11] Glowinski, R. (1984) Numerical methods for nonlinear variational problems, Springer Series in Computational Physics, Springer-Verlag, New York.

[12] Graham J.M.R., Thiriet M. (1990) Pulsatile flow through partially occluded ducts and bifurcations, In "Biomechanical transport processes", Mosora et al. eds., Plenum publishing Corp., N.Y., 59-66.

[13] Grenier, P.A., Beigelman-Aubry, C., Fetita, C., Prêteux, F., Brauner, M.W., Lenoir, S.(2002) New frontiers in CT imaging of airway disease, Eur. Radiol., 12(5), 1022-1044.

[14] Ley, S., Mayer, D., Brook, B.S., van Beek, E.J., Heussel, C.P., Rinck, D., Hose, R., Markstaller, K., Kauczor, H.U. (2002) Radiological imaging as the basis for a simulation software of ventilation in the tracheo-bronchial tree. Eur Radiol., 12(9), 2218-28.

[15] Lorensen, W.E., Cline, H.E. (1987) Marching cubes: A high resolution 3D surface construction algorithm, Computer Graphics, 21(4), 163-169.

[16] Maday, Y., Patera, A.T., Turinici, G., (2002) A priori convergence theory for reduced-basis approximations of single-parameter elliptic partial differential equations, J. Sci. Comput., 17(1-4), 437-446.

[17] Maday, Y., Rønquist, E.M., (2002) A reducedbasis element method, J. Sci. Comput., 17(1-4), 447-459.

[18] Maday, Y. (2003) Some mathematical tools for the modelling of the lung, 21st IFIP TC 7 Conference on System Modeling and Optimization (IFIP'03), Sophia Antipolis, France, July 21-25.

[19] Meyer, F. , Maragos, P. (2000) Nonlinear scalespace representation with morphological levelings, J. Visual Communication Image Representation, 11, 245-265.

[20] Perchet, D., Fetita, C., Vial, L., Prêteux, F., Caillibotte, G., Sbirlea-Apiou, G., Thiriet, M. (2004)
Virtual investigation of pulmonary airways in volumetric computed tomography, Computer Animation Virtual Worlds, 15(3-4), 361-376.

[21] Prêteux, F. (1992) On a distance function approach for gray-level mathematical morphology, in "Mathematical morphology in image processing", Dougherty E. R. (ed.), Dekker.

[22] Pironneau, O. (1982) On the transport-diffusion algorithm and its application to the Navier-Stokes Equations, Numer. Math., 38, 309-332.

[23] Stein, P.D., Walburn, F.J., Blick, E.F. (1980) Damping effect of distensible tubes on turbulent flow: implications in the cardiovascular system, Biorheology, 17(3), 275-281.

[24] Taubin, G., (1995) Curve and surface smoothing without shrinkage, 5 th Int. Conf. on Computer Vision Proc., 852-857.

[25] Taylor, G.I. (1929) The criterion for turbulence in curved pipes, Proc. R. Soc. Lond. A, 124(794), 243-249.

[26] Thiriet, M., Maarek, J.M., Chartrand, D. A., Delpuech, C., Davis, L., Hatzfeld, C., Chang, H. K. (1989) Transverse images of the human thoracic trachea during forced expiration, J. Appl. Physiol., 67, 1032-1040.

[27] Thiriet, M., Issa, R., Graham, J.M.R. (1992) A pulsatile developing flow in a bend, J. Phys. III, 3, 995-1013.

[28] Weibel, E.R. (1963) Morphometry of the human lung, New York, Academic Press. 